\title{
Effect of Different Preparation Conditions On The Properties of Bamboo Fiber-Based Bioactive Composite Membrane
}

Jiang Liuyun ( $\sim$ jlytxg@163.com )

Hunan Normal University https://orcid.org/0000-0002-8592-5736

Bingli Ma

Hunan Normal University

Zhihong Jiang

Hunan Normal University

Yingjun Ma

Hunan Normal University

Yue Wen

Hunan Normal University

Na Zhang

Hunan Normal University

Yan Zhang

Hunan Normal University

Shengpei Su

Hunan Normal University

Xionggui Tang

Hunan Normal University

Xiang Hu

Hunan Normal University

\section{Research Article}

Keywords: Bamboo fiber, nano-hydroxyapatite, composite membrane, degradation

Posted Date: January 3rd, 2022

DOI: https://doi.org/10.21203/rs.3.rs-1185082/v1

License: (c) (1) This work is licensed under a Creative Commons Attribution 4.0 International License.

Read Full License 


\section{Effect of different preparation conditions on the properties of bamboo fiber-based bioactive composite membrane}

Liuyun Jiang*a, b, Bingli Ma a, b, Zhihong Jiang a, b, Yingjun Ma ${ }^{\text {a, b}}$, Yue Wen ${ }^{\text {a, b }}$, Na Zhang a, b, Yan Zhang a, b, Shengpei $\mathrm{Su}^{\mathrm{a}, \mathrm{b}}$, Xionggui Tang ${ }^{*}$, Xiang $\mathrm{Hu}^{*}{ }^{*}$

a National \& Local Joint Engineering Laboratory for New Petro-chemical Materials and Fine Utilization of Resources, College of Chemistry and Chemical Engineering, Hunan Normal University, Changsha 410081, PR China

b Key Laboratory of Chemical Biology \& Traditional Chinese Medicine Research (Ministry of Education, China), College of Chemistry and Chemical Engineering, Hunan Normal University, Changsha 410081, PR China

c College of Physical \& Electronic Science, Hunan Normal University, Changsha 410081, PR China

d State Key Laboratory Developmental Biology of Freshwater Fish, School Life Science, Hunan Normal University, Changsha, 410081, PR China

\section{Abstract}

A novel nano-hydroxyapatite/bamboo fiber (n-HA/BF) bioactive composite membrane was obtained by a simple casting technique. The membrane forming mechanism and the effects of different forming membrane methods, drying methods and n-HA amounts on the properties of n-HA/BF membrane were investigated by Fourier Transform infrared spectroscopy (FT-IR), X-ray diffraction (XRD), scanning electron microscopy (SEM), contact angle, electromechanical universal tester, in vitro soaking in simulated body fluid (SBF) and in vitro cell culture experiment. The results demonstrated that the n-HA dispersity in BF matix was not affected by the prepartion condition, however, the morphologies of membrane was determined by the different preparation conditions owing to different hydrogen bond shrinkage. Moreover, the hydrophilicity of the composite membrane was improved under the condition of the membrane formation in oven, freeze drying and high addition content of n-HA, and the mechanical properties of composite membrane depended on n-HA content. In vitro soaking behavior indicated that the degradability and bone-like apatite deposition could be controled by different preparation conditions. And the cell proliferation experiment showed that the $\mathrm{n}-\mathrm{HA} / \mathrm{BF}$ composite membranes obtained under different preparation conditions were all non-toxic. The above results indicated that the n-HA/BF composite membrane could be obtained by a simple casting technique, and the properties could be controlled by adopting different preparation conditions, which would have a great promising as guide bone tissue regeneration (GBR) membrane, and the study would provide a new application for BF in biomedical field.

Keywords: Bamboo fiber; nano-hydroxyapatite; composite membrane; degradation

\section{Introduction}

Guided bone regeneration (GBR) membrane is commonly used to augment defect area, where

Tel.: +86 07318887 3111; fax: +86 073188873111 ,

E-mail address: jlytxg@163.com, tangxg@hunnu.edu.cn, huxiangsky123123@163.com 
42 the membrane was placed on the bone defect area as barrier membrane to create a singular space, so as to prevent epithelial cells from growing into the defect, and permit the proliferation of osteoblasts and new bone formation for bone tissue regeneration (Jiang et al., 2015; Niu et al., 2021; Li et al., 2020; 2015). Ideally, GBR membrane should possess appropriate mechanical properties, space-retention ability, biocompatibility and biodegradability (Lee et al., 2016; Yu et al., 2020; Hoornaert et al., 2016). To obtain the satisfactory GBR membrane, natural biodegradable polymers has become a research hotspot owing to the better biocompatibility and biodegradability, compared with the synthetic polymers (Prajatelistia et al., 2021; Ma et al., 2019; Mora-Boza et al., 2020; Bierhalz and Moraes, 2017; Pappu et al., 2019; Gurunathan et al., 2015; Weng et al., 2020).

Bamboo fiber $(\mathrm{BF})$ is a natural fiber extracted from bamboo, which has high strength, biodegradability and low cost. Therefore, BF is usually used as a reinforcing agent for polymers (Khalil et al., 2012; Liu et al., 2012; Phuong et al., 2019; Long et al., 2019; Zuo et al. 2019). In our group previous study, we have systematically studied and concluded that BF had remarkable reinforce effect on the n-HA/poly (lactide-co-glycolide) (n-HA/PLGA) composite (Li et al., 2015; Jiang et al., 2017; 2018; 2019). Moreover, BF has been used to reinforce membrane by electrospinning (Chingakham et al., 2020; Cai et al., 2018). In viewing of the fact that the casting membrane had denser structure and simple preparation method, which would display better mechanical strength and slower degradation than electrospinning membrane (Oksana et al., 2020). Therefore, we studied the reinforce effect of carboxylated BF on chitosan-based casting membrane via ionic crosslinking (Tang et al., 2020). However, the preparation procession of carboxylated $\mathrm{BF}$ was too tedious. In addition, in the aboved study, BF was usually added in the 
64 primeval state fiber form as reinforcement, which would be adverse for dispersion, so it would be expected to explore a novel polymer membrane by a simple and green processing.

BF could be dictrectly dissolved into homogeneous solution and obtained casting membrane, whileas, as we know, pure BF membrane lacks osteoconductivity, which would be detrimental to guide bone tissue regeneration, while nano-hydroxyapatite (n-HA) was similar to with inorganic component of natural bone, so it was normally added into polymeric matrix as nanofiller to endow polymers with better biological performance (Zhao et al., 2018; Muhammad et al., 2017). In our previous study, we found BF could replace other polymers to develop n-HA-based nanocomposite by co-precipitation method, and it displayed a promising to be used as bone materials (Ma et al., 2020). However, similarly, whether BF could be replayed other polymers to obtain n-HA/BF composite membrane by solution blending method, and whetherthe different prepartion conditions including different forming membrane methods, drying methods and n-HA amounts would bring about different effect on the properties of n-HA/BF membrane, and whether it would be used as GBR membrane, which were all not be reported and it was worth exploring.

Based on these, in this work, we attempt to study the fabrication of the n-HA/BF composite membrane by casting method, and the effects of different preparation conditions on the properties of the n-HA/BF composite membranes, including forming film methods (in air and in oven), different drying methods (in air, in oven, in freeze dryer) and different n-HA content $(10 \%, 20 \%$, $30 \%$ and $40 \%$ ), were studied by Fourier Transform infrared spectroscopy (FT-IR), X-ray diffraction (XRD), scanning electron microscopy (SEM), contact angle, electromechanical universal tester, Moreover, the degradation behavior of the composite membrane was 
86 investigated by soaking in simulated body fluid (SBF). Finally, the cell culture experiment was

87 carried out. The main purpose of the work is to demonstrate the feasibility of the fabrication of the $\mathrm{n}-\mathrm{HA} / \mathrm{BF}$ composite membrane by a simple casting method, so as to provide a new GBR membrane by making full use of natural biomass resources.

\section{Experiment Section}

\section{Materials}

92 Bamboo fiber (BF) was provided by Zhejiang A\&F University, whose size was $\Phi(0.03-0.2) \mathrm{mm}$ $93 \times(6-10) \mathrm{cm}$. Dimethylacetamide $(\mathrm{DMAc}, \mathrm{AR})$ and $\mathrm{LiCl}(\mathrm{AR})$ were purchased from Aladdin. $\mathrm{Ca}$ $94\left(\mathrm{NO}_{3}\right)_{2} \cdot 4 \mathrm{H}_{2} \mathrm{O}(\mathrm{AR}), \mathrm{Na}_{3} \mathrm{PO}_{4} \cdot 12 \mathrm{H}_{2} \mathrm{O}(\mathrm{AR}), \mathrm{P}_{2} \mathrm{O}_{5}$, and $\mathrm{NaOH}(\mathrm{AR})$ were all from Tianjin Fengchuan 95 Chemical Reagent Technologies Co. Ltd.. Other agents were analytical grade.

96

\section{Preparation of the $\mathrm{n}-\mathrm{HA} / \mathrm{BF}$ composite membranes}

Bamboo fiber was dissolved in DMAc with the concentration of $1.3 \mathrm{wt} \%$. A certain amount of n-HA was dispersed in DMAc by ultrasonic treatment, which was slowly added into bamboo fiber solution with the magnetic stirring, and the evenly dispersed mixture solution was poured on the clean and dry glass plate, and the glass rod with two copper rings was applied to control the thickness of the membrane. Then, the glass plate covered with n-HA/BF mixture solution was put in air or in oven to form film, afterword, it was dried in air, in oven or freeze drying, thus, n-HA/BF composite membrane with $20 \%$ n-HA were obtained by six methods, which was noted as air-air, air-oven, air-freeze drying, oven-air, oven-oven and oven-freeze drying, respectively. Moreover, n-HA/BF composite membrane obtained by air-air method with different n-HA contents of $10 \%, 20 \%, 30 \%$ and $40 \%$ n-HA were prepared in the similar procedure.

\section{Characterization of the composite membranes}


108 The appearance of membranes obtained by the two different forming membrane methods of

109 air-oven and oven-oven were given as examples, which was taken by normal digital camera.

110 Thermo Niclet 670 spectrometer was used to analysis the Fourier transformation infrared

111 (FTIR) of samples, and the collected spectrum range was $600 \sim 4000 \mathrm{~cm}^{-1}$.

112 The phase analyses of samples were carried out by X-ray diffraction (XRD) (a Rigaku

113 Corporation $\mathrm{X}$-ray diffractometer) with $\mathrm{Cu}-\mathrm{K} \alpha$ radiation, the scanning speed of $5^{\circ} / \mathrm{min}$ at $40 \mathrm{kV}$

114 and $45 \mathrm{~mA}$, the range of $2 \theta=10 \sim 70^{\circ}$.

115 The morphologies of samples treated with the gold sputtering were observed by scanning

116 electron microscopic (SEM, S-520, Hitachi, Japan).

117 The contact angles of samples were measured with Rotating drop interfacial tensiometer

118 (TX500TM, Kono, USA). The sample was put on the slide, and the distilled water was dropped

119 onto the surface of the liquid by stop drop method, then the water drop on the sample was

120 observed.

121 The tensile strengths of samples with the size of $0.2 \mathrm{~mm} \times 4 \mathrm{~mm} \times 60 \mathrm{~mm}$ were measured by

122 electromechanical universal testing machine (WDW-20, China) with the speed of $5 \mathrm{~mm} / \mathrm{min}$ with

$12360 \%$ relative humidity at room temperature, and the mean value was calculated based on the five

124 parallel samples of each specimen.

125 In vitro degradation of the composite membranes

126 The degradation of $\mathrm{n}-\mathrm{HA} / \mathrm{BF}$ composite membrane in vitro was studied by soaking in SBF,

127 whose ion concentration was very similar to that of human plasma, and it was obtained according

128 to the following procedure, that is, $\mathrm{NaHCO}_{3}(0.350 \mathrm{~g}), \mathrm{NaCl}(7.996 \mathrm{~g}), \mathrm{KCl}(0.224 \mathrm{~g}), \mathrm{K}_{2} \mathrm{HPO}_{4}$.

$1293 \mathrm{H}_{2} \mathrm{O}(0.228 \mathrm{~g}), \mathrm{CaCl}_{2}(0.278 \mathrm{~g}), \mathrm{Na}_{2} \mathrm{SO} 4(0.071 \mathrm{~g}), \mathrm{MgCl}_{2} \cdot 6 \mathrm{H}_{2} \mathrm{O}(0.305 \mathrm{~g})$ were dissolved in 
130 deionized water in order, and buffer with trimethylolmethylaminomethane $(6.057 \mathrm{~g})$ and

131 hydrochloric acid to adjust the solution to physiological $\mathrm{pH} 7.40$ at $37^{\circ} \mathrm{C}\left( \pm 0.5^{\circ} \mathrm{C}\right)$. The samples

132 were taken out from SBF at 2, 4, 6 and 8 weeks, and filter paper was used to absorb surface

133 residual washing water. The water absorption and water loss were as follows.

Weight loss rate $/ \%=\left(\mathrm{w}_{1}-\mathrm{w}_{3}\right) / \mathrm{w}_{1} \times 100 \%$

Water absorption $/ \%=\left(\mathrm{w}_{2}-\mathrm{w}_{3}\right) / \mathrm{w}_{3} \times 100 \%$

139 as $\mathrm{W}_{2}$ and $\mathrm{W}_{3}$, after being entirely dried after soaking, respectively.

140 In vitro cell biocompatibility of the composite membranes

141 Bone mesenchymsal stem cells (BMSCs) were used to primarily assess in vitro cells viability,

142 which was isolated according to the related literature (Hoseini et al., 2015; Ye et al., 2019). The

143 samples with the thickness of $0.2 \mathrm{~mm}$ and diameter of $6.0 \mathrm{~mm}$ were cleaned with $75 \%$ ethanol

144 solution, sterilized under ultraviolet lamp. The treated samples were placed in a 96-well plate

145 with the density of 4000 cells/well without disturbed in an incubator for 3 hours, then an

146 additional $1 \mathrm{~mL}$ culture medium was added into each well.

147 The cell proliferation was evaluated by MTT (3-(4,5-dimethylthiazol-2yl)-2,5-diphenyl-2H-

148 tetrazolium bromide) assay (Priyadarshini et al., 2018; Shakeri et al., 2014; Hivechi et al., 2021).

149 At designated time of 1, 2 and 3 days, the medium for the cell-seeded materials were discarded,

150 and $100 \mu \mathrm{L}$ MTT solution with $3 \mathrm{mg} / \mathrm{mL}$ was added, incubated at $37^{\circ} \mathrm{C}$ in an air atmosphere

151 containing $5 \% \mathrm{CO}_{2}$ for at least 4 hours, and $100 \mu \mathrm{L}$ DMSO was added to dissolve the formazan

152 crystals. Then the $200 \mu \mathrm{L}$ purple solution was absorbed and transferred into a new 96-well plate,

153 and the optical density (OD) values of the solution were measured in microplate reader (Synergy 
HTX, BIOTEK, USA) at $495 \mathrm{~nm}$.

\section{Results and discussion}

\section{Characterization of surface-modified n-HA}

157 Appearance of the membranes

158 Fig. 1 displays the appearance of membrane, it can be seen that the membrane formed in air had

159 smaller area and thicker than that formed in oven, which was mainly caused from the different

160 hydrogen bonds between the bamboo fiber molecules. In air, the bamboo fiber was easy to 161 absorb the water, which would make the hydrogen bond shrinkage in the bamboo fiber, thus the

162 bamboo fiber was gelled and $\mathrm{DMAC} / \mathrm{LiCl}$ solution precipitated from the edge, resulting in 163 thicker film and smaller area. While in oven, DMAC solvent was easy to volatilize and the 164 hydrogen bond between the bamboo fiber molecules was destroyed, and the bamboo fiber has 165 been formed before shrinkage, resulting in thinner film and larger area.

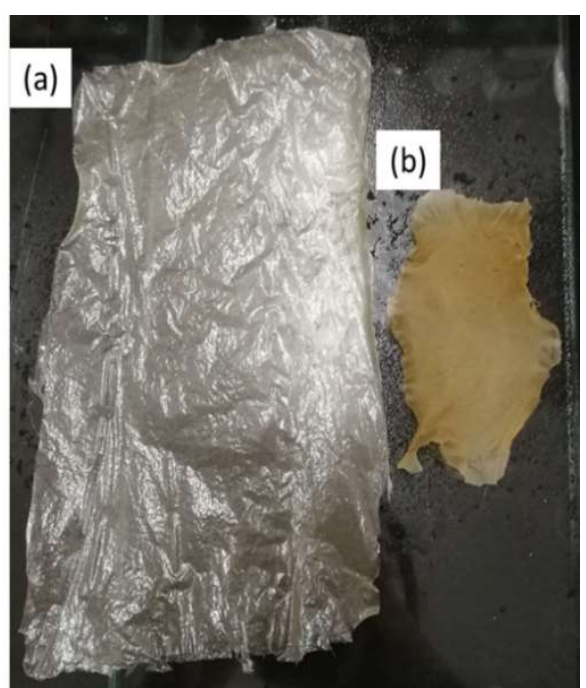

Fig.1. Membrane appearances formed by different conditions. (a) in oven, (b) in air.

173 IR analysis

174 Fig. 2 is the FT-IR spectra of different n-HA/BF composite membrane obtained by different

175 methods and different n-HA contents. The characteristic peaks of $2920 \mathrm{~cm}^{-1}$ and $2846 \mathrm{~cm}^{-1}$ 

composite membrane. In addition, the characteristics peaks at $3567.2 \mathrm{~cm}^{-1}$ attributed to the tensile vibration of $\mathrm{OH}^{-}$and the peaks at $1095 \mathrm{~cm}^{-1}, 604 \mathrm{~cm}^{-1}$ and $565 \mathrm{~cm}^{-1}$ of $\mathrm{PO}_{4}{ }^{3}$-were related to n-HA (Chesley,et al., 2020). The peak position did not transfer obviously when n-HA was added into BF, which indicated that n-HA was simply blended with BF without chemical change. Compared with the membrane formed in oven and in air, the intensity of the peak at $1044 \mathrm{~cm}^{-1} \mathrm{of}$ the film formed in oven was markedly weakened, which was caused by less substance in the unit area for the thinner film formed in the oven. Additionally, for the different n-HA/BF composite membrane with different n-HA contents, there was no obvious difference for the characteristics

185 peak.

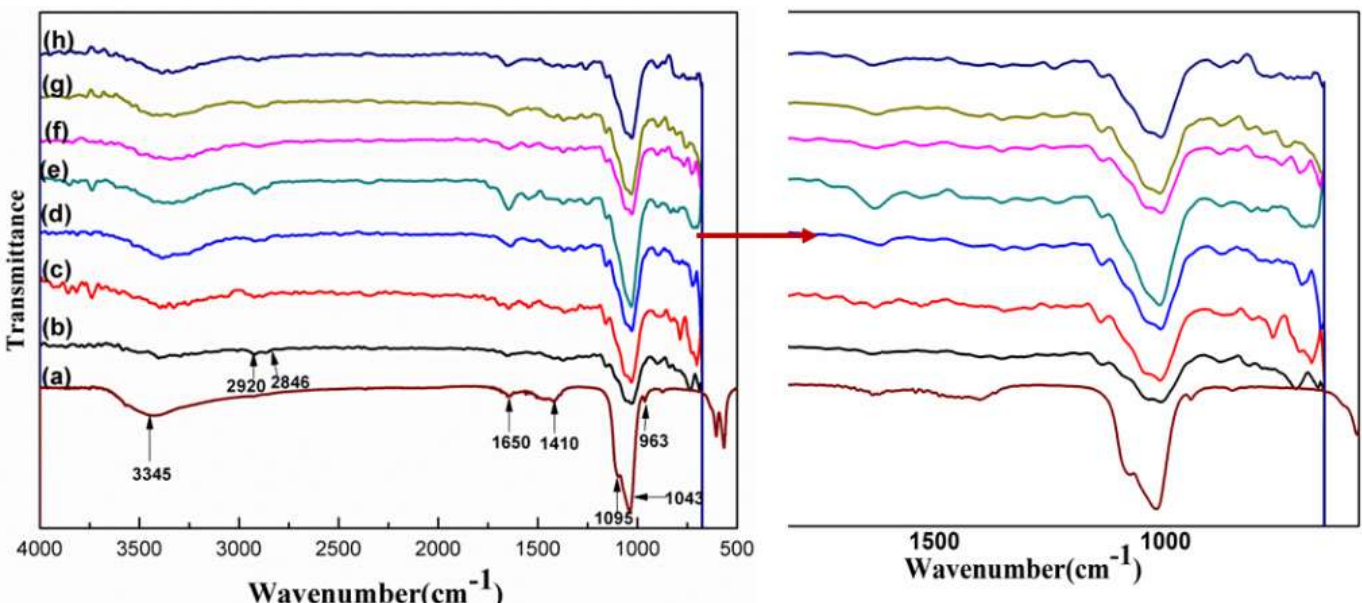

Wavenumber $\left(\mathrm{cm}^{-1}\right)$

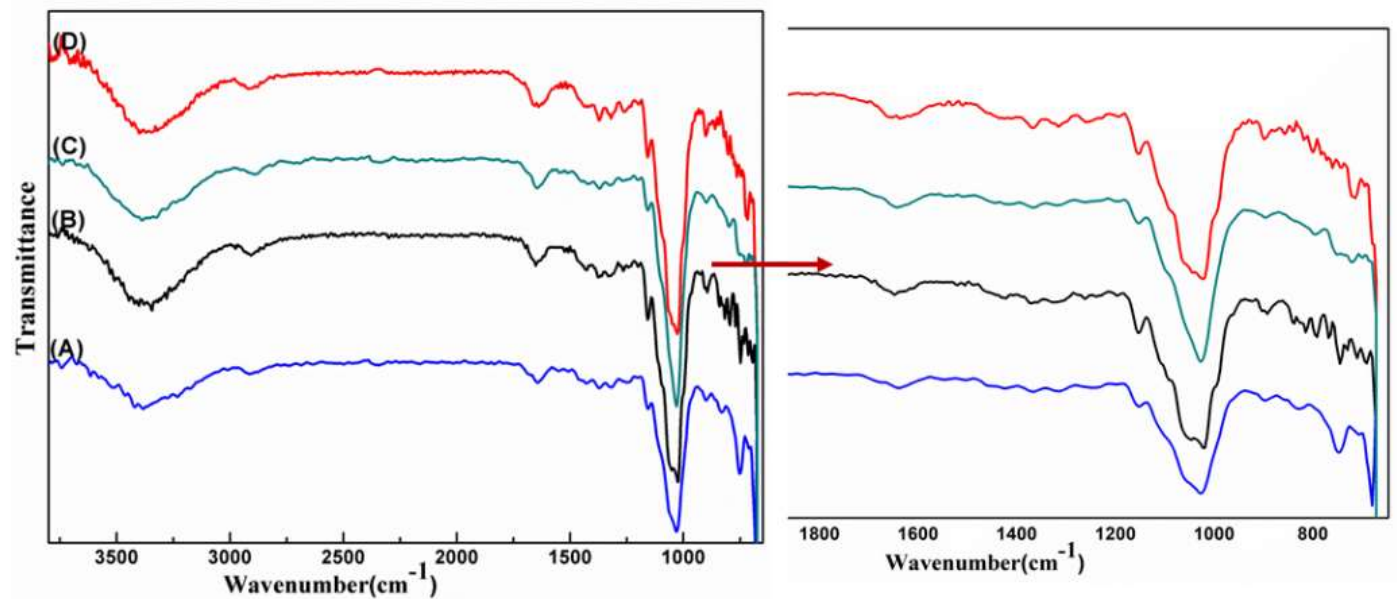

Fig.2. FTIR spectra of samples. (a)n-HA, (b)BF, (c)Air-Air, (d)Air-oven, (e)Air-freeze drying, (f) Oven-air, (g)Oven-oven, (h)Oven-freeze drying, (A)10\% n-HA/BF, (B)20\% n-H $\mathrm{A} / \mathrm{BF},(\mathrm{C}) 30 \% \mathrm{n}-\mathrm{HA} / \mathrm{BF}$, (D) $40 \%$ n-HA/BF. 
XRD investigation

To further understand the phase structure of n-HA/BF membrane, XRD pattern is shown in Fig. 3. Obviously, the amorphous peak at $20.5^{\circ}$ was the peak of bamboo fiber (Guimaraes, et al., 2015), marked with “»", and the peaks at $25^{\circ}$ and $31^{\circ}$ of n-HA were found, noted as " $"$ ". The crystallization peak position of n-HA in the composite membrane did not change, indicating that the two components of n-HA and BF were only blended. Similarly, the crystallization peaks of Fig.3 (f), (g), (h) of n-HA were also obviously weaker than those of Fig.3 (c), (d) and (e) formed in air. This was because the content of n-HA in the membrane formed in the oven was less than that in the membrane formed in the oven, which leads to the weak crystal peak in the spectrum.

With the increase of n-HA content, the characteristic peak and its crystallinity of n-HA in different membrane increased, which also confirmed that n-HA and BF were only blended without chemical reaction.
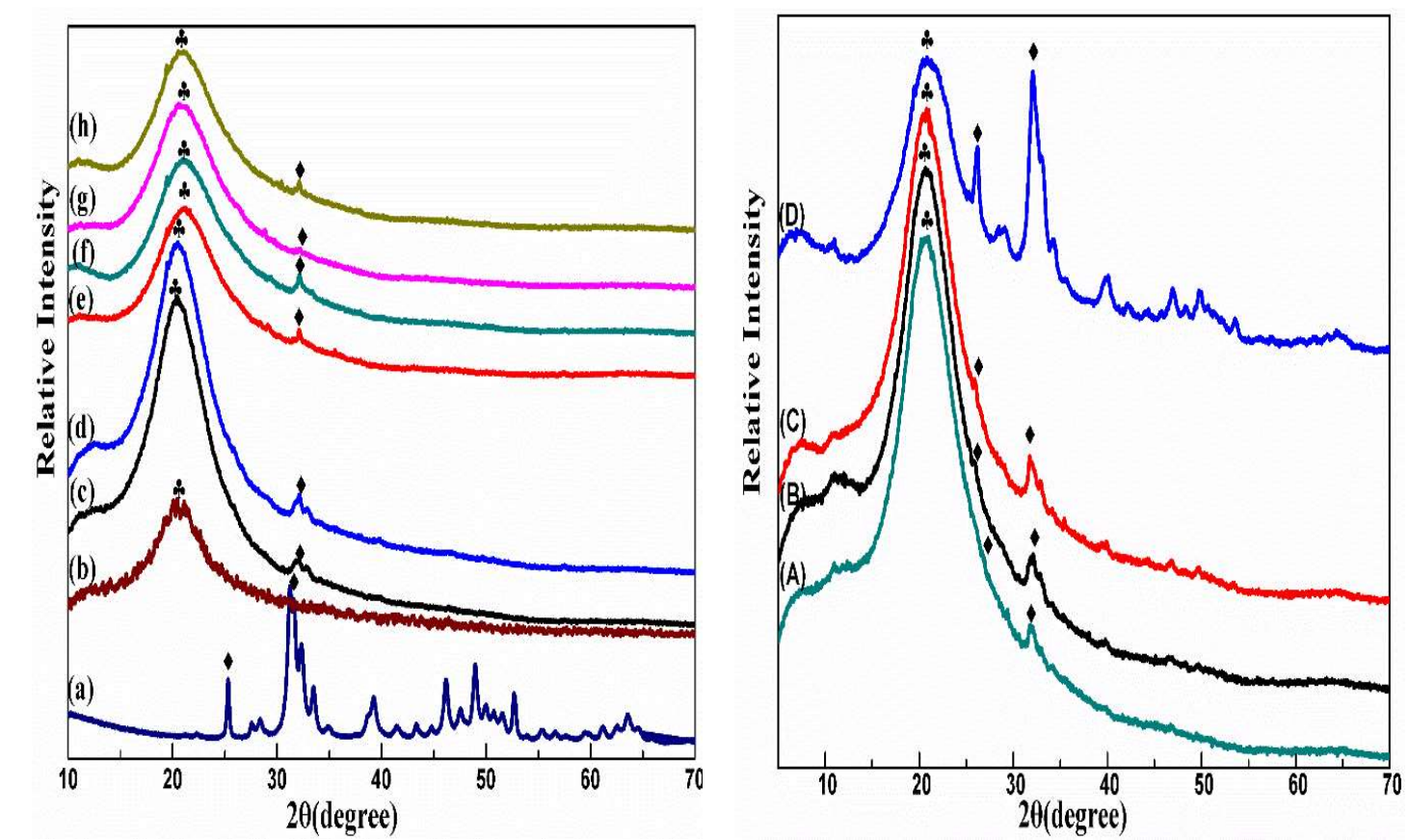

Fig. 3 XRD of samples. (a) n-HA, (b)BF, (c) Air-Air, (d) Air-oven, (e) Air-freeze drying, (f) Oven-air, (g) Oven-oven, (h) Oven-freeze drying, (A) 10\% n-HA/BF, (B) $20 \%$ n-HA/BF, (C) 30\% n-HA/BF, (D) 40\%n-HA/BF. 
SEM observation

222 Fig. 4 shows the morphologies of n-HA/BF composite membranes obtained by different

223 methods. From the SEM micrographs, it can be seen that white particles existed in the composite

224 membranes, which showed the existence of n-HA. However, there was subtle difference for the

225 dispersion of n-HA particles in the membrane, and the n-HA was relatively more uniform in oven

226 (Fig.4(d), (e), (f)) than in air (Fig.4(a), (b), (c)), which was caused by the different hydrogen

227 bond between molecules during the forming membrane of bamboo fiber. In oven, the solvent of

228 DMAC was evaporated at high temperature, and the fiber morphology had been fixed before the

229 hydrogen bond between bamboo fiber molecules shrinking, which would bring out better

230 dispersion of $n-H A$.

231

232
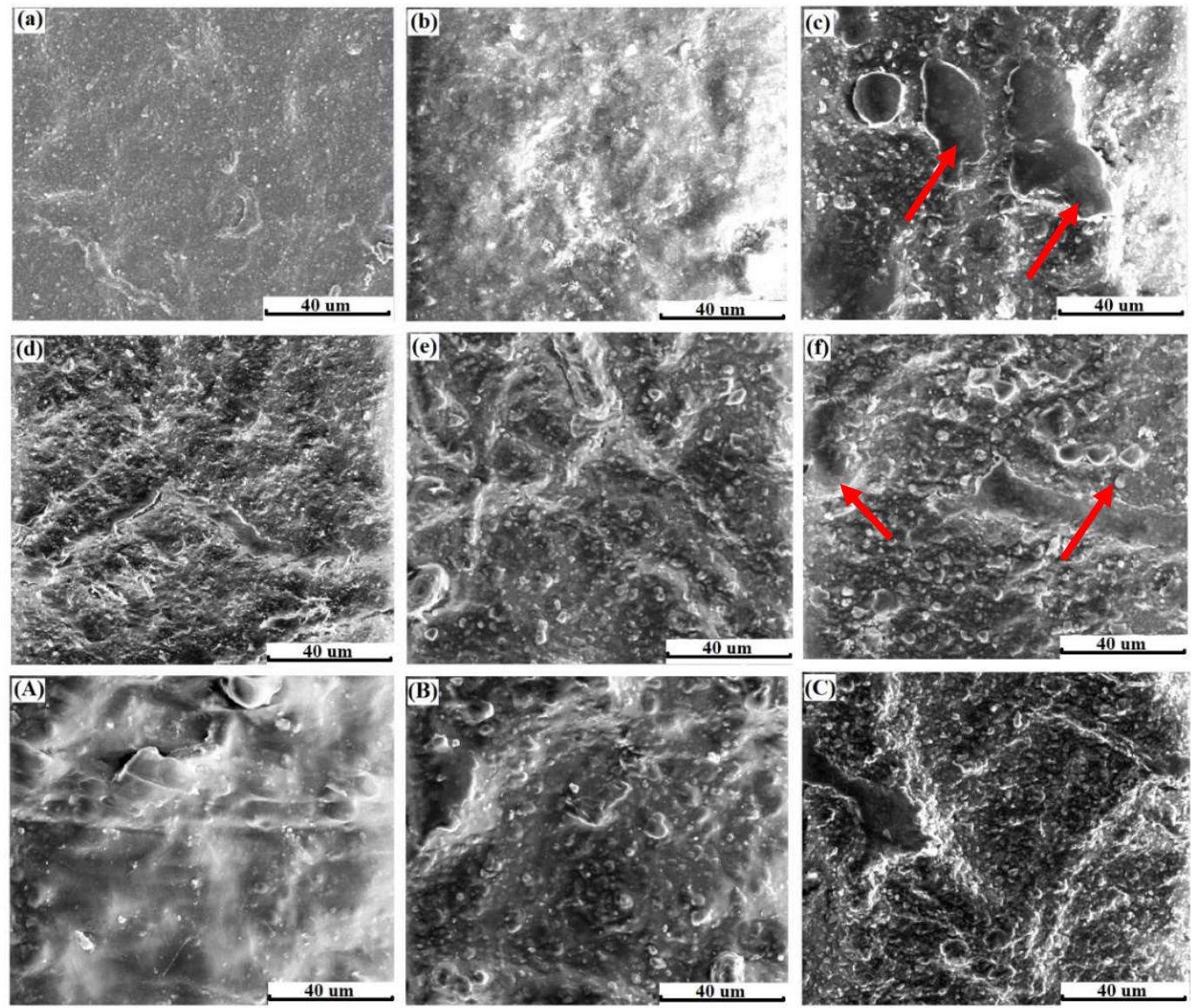

Fig. 4 SEM of samples. (a)Air-Air, (b)Air-oven, (c)Air-freeze drying, (d)Oven-air, (e)Oven-oven, (f)Oven-freeze drying, (A)10\%HA/BF, (B) 30\%HA/BF, (C)40\%HA/BF.

However, for the three different dry methods, the surfaces were compact without porous 
244 structures, and there was no obvious difference for the surface in air and in oven, which indicated

245 that drying method in air or in oven had little effect on the membrane morphology after bamboo

246 fiber molding, and the compact structure could effectively prevent the invasion of connective

247 tissue. While for the freeze-drying method, there was some closed pores on the surface of

248 freeze-dried membrane (Fig.4(c), (f)), which was originated from the pores left by the

249 sublimation of water molecules, and the porous structure was conducive to cell adhesion for

250 guided bone regeneration. Additionally, for the composite membranes with different n-HA

251 contents, the white particles gradually increased on the surface of the membrane, but there was

252 no obvious agglomeration phenomenon, and there displayed excellent interface between n-HA

253 and BF, which indicated that the hydrophilic n-HA and BF had good compatibility.

254 Contact angle measurement
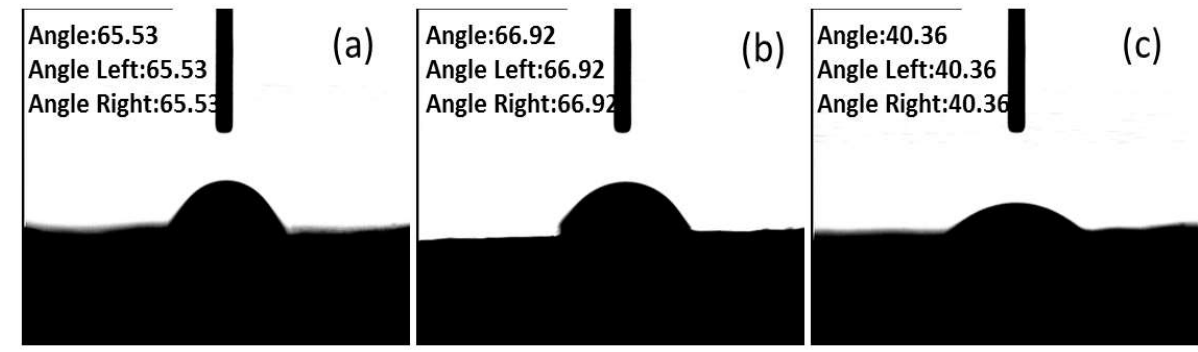

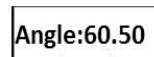

Angle Left:60.50

Angle Right: 60.50

260

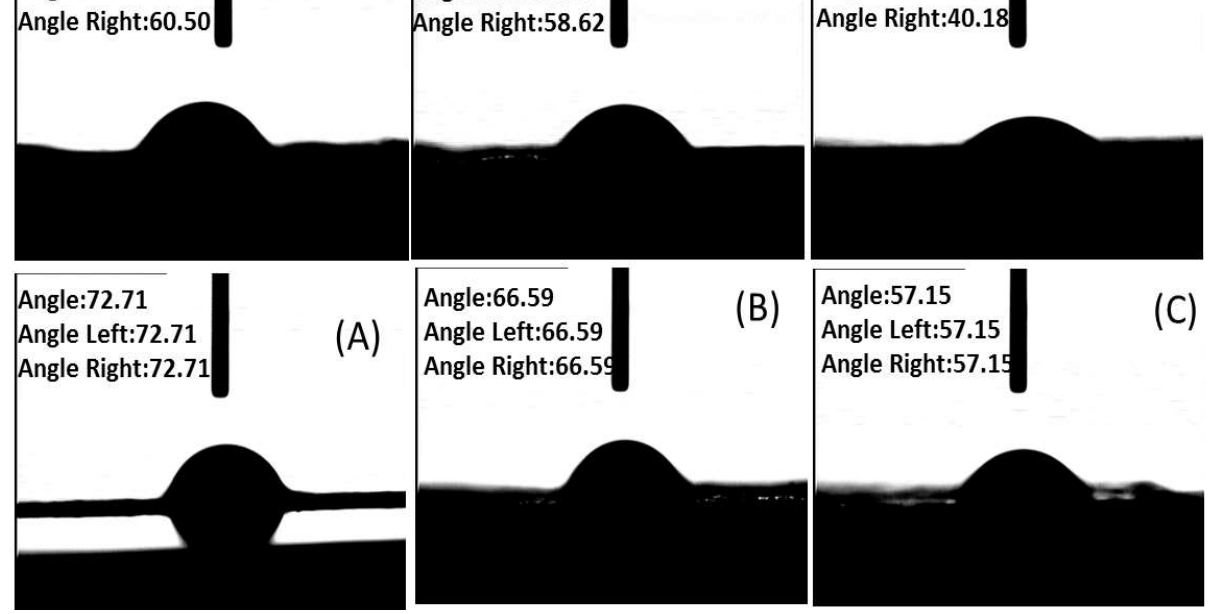

Fig.5. Contact angle of the n-HA/BF composite membranes obtained by different methods. (a) Air-Air, (b) Air-oven, (c) Air-freeze drying, (d) Oven-air, (e) Oven-oven, (f) Oven-freeze drying, (A) 10\%-n-HA/BF, (B) 30\% n-HA/BF, (C) 40\% n-HA/BF. 
To further make clear the hydrophilicity of the n-HA/BF composite membrane, the contact

267 angle of the n-HA/BF composite membranes were tested with rotating drop interfacial 268 tensiometer by dropping distilled water onto the liquid surface, and the results are shown in Fig.

269 5. As expected, the contact angle values of all $\mathrm{n}-\mathrm{HA} / \mathrm{BF}$ casting membranes were less than $90^{\circ}$, 270 which proved that $\mathrm{n}-\mathrm{HA} / \mathrm{BF}$ casting membranes were hydrophilic membrane. Comparing with 271 the membranes obtained by different methods, we found that the membranes obtained by the 272 same forming membrane method had a similar contact angle value, while the freeze-dried 273 membrane possessed lower contact angle because of the rough membrane surface with the 274 porous structure (Fig.5(c)), and the stronger hydrophilicity would be more useful for cell 275 adhesion and proliferation (Dhinasekaran, et al., 2021). However, for the same dried method, the 276 membrane formed in oven displayed lower contact angle than that of the membrane formed in 277 air, and the reason was that the hydrogen bond between the film-forming molecules was 278 destroyed and the surface tension was reduced when the membrane was dried in oven. In 279 addition, for the $\mathrm{n}-\mathrm{HA} / \mathrm{BF}$ composite membranes with different $\mathrm{n}-\mathrm{HA}$ contents, the contact angle 280 gradually decreased with the increasing of n-HA, which showed that the higher n-HA content 281 brought about better hydrophilicity of the membrane.

\section{Tensile strength test}

283 Fig. 6 shows the tensile strengths of the n-HA/BF composite membranes obtained by different 284 methods. We found that membrane formed in air had higher mechanical strength than that in 285 oven. Comparing to the membrane formed in air (Fig.6 (a), (b), (c)), the tensile strength of 286 membrane dried by freeze-drying was the worst, and the tensile strength of the membrane dried 287 in air was improved by $69.64 \%$, and dried in oven was increased by $51.7 \%$ than dried by 
freeze-drying, respectively. Likely, for the three membranes formed in oven (Fig.6(d), (e) and

289 (f)), the tensile strengths of the membranes dried in air and in oven were improved by $23.89 \%$

290 and 5\%, respectively. The reason was that the freeze-drying membrane was porous structure, and

291 the forming membrane or dry membrane in oven caused the destruction of intermolecular

292 hydrogen bond during molding, resulting in thinner membrane and larger area, so as to display

293 lower tensile strength. For the n-HA/BF composite membranes with different n-HA contents, the

294 tensile strength increased at first but decreased with the increasing of n-HA content, and the $20 \%$

295 n-HA/BF composite membrane displayed the highest tensile strength, which was accord with the nanoparticles filler reinforce principle (Yadav, et al., 2020), and the mechanical strength could meet the application requirement of GBR membrane (Castro, et al., 2018).

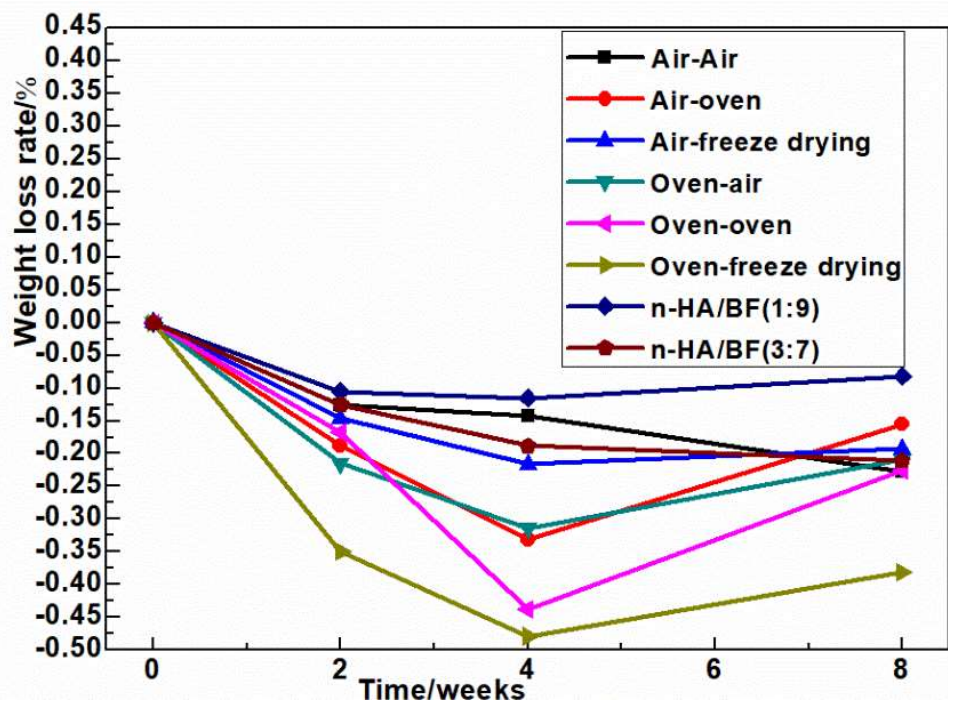

Fig.7 Weight loss rate of samples in SBF.

\section{In vitro degradation and cell culture}

307 Weight loss of composite membrane after degradation

308 Fig.7 shows the weight loss rate of n-HA/BF composite membrane. It can be seen that the weight 309 loss rate of the composite membrane first decreased and then increased within 8 weeks. During 
310 the first 4 weeks, the weight loss rate was negative continuously, which indicated that the mass of

311 composite membrane did not decrease but increased after immersion, meaning that the amount of

312 apatite deposited on the surface of the membrane was greater than the mass of degradation, so

313 that the total mass of composite membrane was greater than that before immersion. Similarly, the

314 weight loss rate of 4-8 weeks was negative, but showed an upward trend, indicating that the

315 membrane possessed greater degradation. Comparing to the different composite membranes, the

316 weight loss rate of the membrane obtained by oven-freeze drying method changed the most

317 among the membrane different methods, which indicated that bone like apatite adhered most

318 owing to the porous structure, and the much apatite deposition would have better biological

319 activity. Moreover, for the composite membranes with different n-HA contents, the higher the

320 content of $n-H A$, the greater the negative value of weight loss rate, indicating that the total

321 weight was more than the original weight, which was mainly caused by the more new apatite

322 deposited, and it was further proved that the higher the content of n-HA was more favorable for

323 the bone-like apatite deposition (Kumar et al., 2014).

324 Water absorption of composite membrane after degradation

325 Fig.8 gives the water absorption of n-HA/BF composite membrane. The results showed that the

326 water absorption of $\mathrm{n}-\mathrm{HA} / \mathrm{BF}$ composite membrane had a similar trend during the immersion

327 process, that is, the water absorption increased slightly at the initial degradation, and then

328 decreased greatly from 2 weeks to 4 weeks, which was caused by the bone-like apatite deposition

329 with the extension of soaking time. Moreover, the freeze-dried membrane displayed the lowest

330 water absorption, and the main reason was that the porous structure had the fastest degradation,

331 which would produce much micropore and bring more apatite deposition, and it could be proved 
by the weight loss result. Similarly, among the composite membrane materials with different n-HA content, the higher BF content, the higher water absorption showed, which was related to

334 the water absorption of BF. In a word, the water absorption results indicated that the n-HA/BF

335 composite membrane had good degradation and water absorption performance.

SEM observation of composite membrane after degradation

Fig. 9 shows the surface morphology of n-HA/BF composite membrane after soaking in SBF for 8 weeks. It can be found that new bone like apatite was produced on the surface of the n-HA/BF composite membrane. However, comparing to the different composite membranes, the composite membrane formed in oven had less bone like apatite (seen Fig.9 (a), (b), (c)) than the membrane formed in air (seen Fig.9 (d), (e), (f)). The reason was that the hydrogen bond between molecules in cellulose shrank, and n-HA particles were wrapped in the inner part of the membrane when the

352 membrane was formed in oven. While the composite membrane was formed in the oven, the 353 intermolecular hydrogen bond was broken, and n-HA particles were dispersed on the surface of 
354 the membrane, which was more conducive to the deposition of bone-like apatite on the surface of

355 the membrane, and the bone-like apatite would help to improve interface between the

356 tissue-implant and its surrounding tissues (Zhu, et al., 2020).

357 For the composite membrane with different n-HA contents of $10 \%, 30 \%$ and $40 \%$ n-HA

358 (seen Fig.9(A), (B), (C)), respectively. From the 10\% n-HA/BF composite membrane, it can be

359 seen that there were pores on the surface of bamboo fiber (Fig.9(A)), which indicated that

360 bamboo fiber could be degraded during soaking. With the increase of n-HA content, the more

361 bone-like apatite was deposited, which further demonstrated that the n-HA in composite

362 membrane had good induce bone like apatite deposition, and the results were consistent with the

363 previous analysis.

364

365

366

367

368

369

370
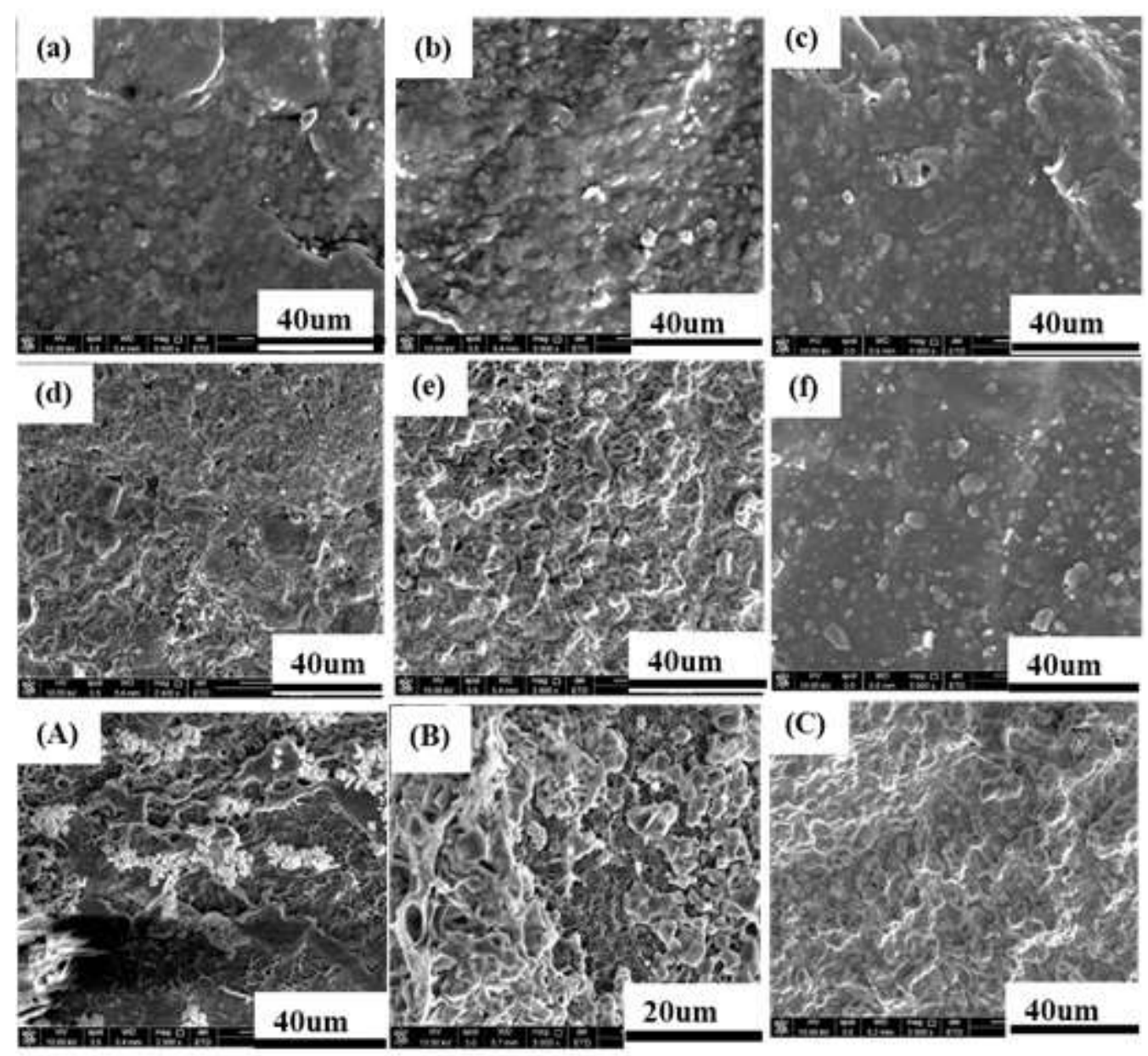

Fig. 9. SEM micrographs of samples after soaking for 8 weeks. (a) Air-Air, (b) Air-oven, (c)Air-freeze drying, (d) Oven-air, (e) Oven-oven, (f) Oven-freeze drying, (A)10\% n-HA/BF, (B) $30 \%$ n-HA/BF, (C) $40 \%$ n-HA/BF. 
377 The results of MTT cell proliferation of BMSC cultured on the surface of n-HA/BF composite 378 membrane for 1, 2 and 3 days are shown in Fig. 10. It can be seen that the OD value of each 379 sample increased significantly with the extension of culture time, indicating that cells could normally grow and proliferate on different membrane surfaces. In addition, the OD values of

381 freeze-dried composite membrane were significantly higher than those of the membrane dried in 382 air or in oven, which further indicated that the porous structure of freeze-dried composite 383 membrane was more conducive for cell proliferation and had good biocompatibility (Ai, et al., 384 2021). Comparing to the n-HA/BF membranes with $10 \%, 20 \%$ and $30 \%$, the OD value enhanced 385 significantly with the increase of n-HA content and the extension of culture time. The results 386 showed that the cells proliferated rapidly on the surface of the membrane, suggesting that the 387 n-HA/BF composite membrane had good biological properties (Bee et al., 2019). This was 388 consistent with the previous in vitro immersion analysis.

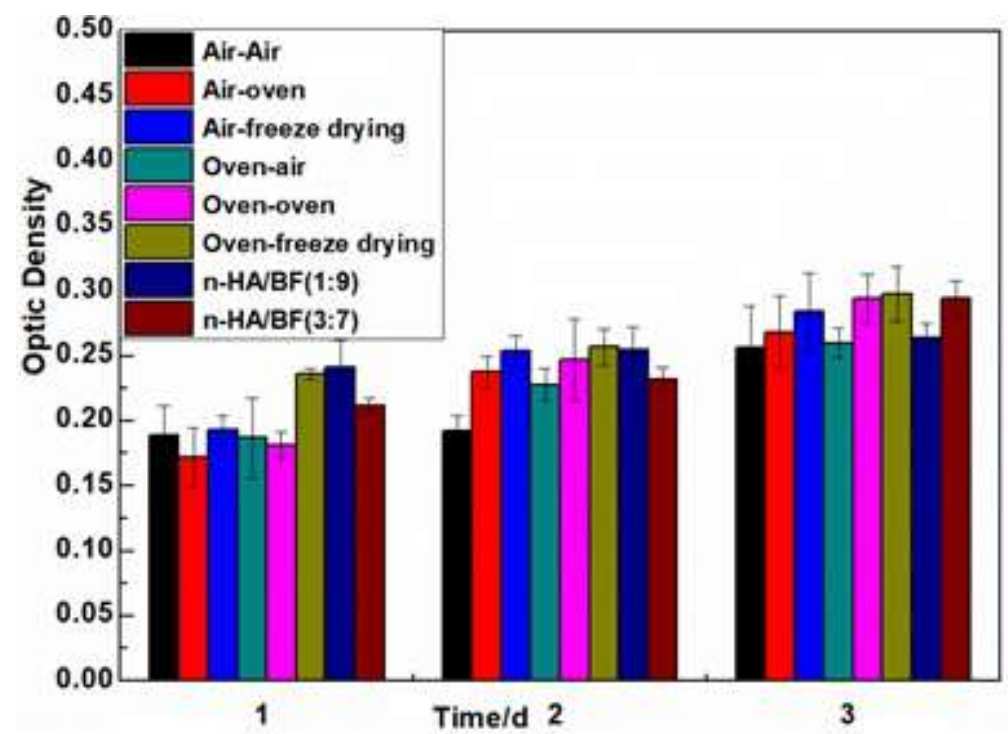

Fig. 10. MTT value of cell culture on sample surface.

\section{Conclusions}


398 In this study, the n-HA/BF composite membrane was successfully prepared by a simple casting 399 technology, and the effects of different forming membrane, drying methods, and different n-HA 400 contents on the composite membrane were studied. The results confirmed that there was no 401 chemical bonding between n-HA and BF components, however, the membrane formed in air was 402 thicker than that in oven, because the hydrogen bond shrinkage made the BF gel and $403 \mathrm{DMAC} / \mathrm{LiCl}$ solution precipitated from the edge in air. Thus, the tensile strength of the 404 membrane formed in air were higher than that of in oven, especially, the $20 \% \mathrm{n}-\mathrm{HA} / \mathrm{BF}$ 405 composite membrane was the highest, but the tensile strength of freeze-dried composite 406 membrane was poorest because of the porous structure. The contact angle test of the composite 407 membrane showed that the composite membrane was hydrophilic, and the freeze-dried 408 membrane brough better hydrophilicity owing to the rough surface and the small surface tension. 409 SBF soaking results indicated that n-HA/BF composite membrane displayed different 410 degradability, and the higher n-HA content in composite membrane displayed better bone-like 411 apatite deposition. The cell proliferation results showed that the composite membrane had no 412 cytotoxicity. This study would provide a new way for developing a novel GBR membrane based 413 on the utilization of natural BF.

\section{Acknowledgement}

415 The authors would like to thank the support of the Natural Science Foundation of Hunan 416 province (2020JJ4430), and Key projects of Hunan Provincial Department of Education 417 (20A315).

\section{References}

419 Ai CC, Liu L, Goh JCH (2021). Pore size modulates in vitro osteogenesis of bone marrow mesenchymal stem 
cells in fibronectin/gelatin coated silk fibroin scaffolds. Mat Sci Eng. C-Mater.124: 112088.

Bee SL, Hamid ZAA (2019). Characterization of chicken bone waste-derived hydroxyapatite and its functionality on chitosan membrane for guided bone regeneration. Compos Part B-Eng. 163: 562-573.

Bierhalz ACK, Moraes AM (2017). Composite membranes of alginate and chitosan reinforced with cotton or linen fibers incorporating epidermal growth factor. Mat Sci Eng. C-Mater. 76: 287-294.

Castro AGB, Diba M, Kersten M, Jansen JA,van den Beucken JJJP, Yang F (2018). Development of a PCL-silica nanoparticles composite membrane for Guided Bone Regeneration. Mat Sci Eng. C-Mater. 85: $154-161$.

Chesley M, Kennard R, Roozbahani S, Kim SM, Kukk K, Mason M (2020). One-step hydrothermal synthesis with in situ milling of biologically relevant hydroxyapatite. Mat Sci Eng. C-Mater. 113: 110962.

Chingakham C, Manaf O, Sujith A, Sajith V (2020). Hydrophobic nano-bamboo fiber-reinforced acrylonitrile butadiene styrene electrospun membrane for the filtration of crude biodiesel. Appl Nanosci. 10: 795-806.

Cai J, Zhou R, Li TT, He JR,Wang GZ, Wang HB, Xiong HG (2018). Bamboo cellulose-derived cellulose acetate for electrospun nanofibers: synthesis, characterization and kinetics. Cellulose 25: 391-398.

Dhinasekaran D, Vimalraj S, Rajendran AR, Saravannan S, Purushothaman B, Subramaniam B (2021). Bio-inspired multifunctional collagen/electrospun bioactive glass membranes for bone tissue engineering applications. Mat Sci Eng. C-Mater. 126: 111856.

Guimaraes M, Botaro VR, Novack KM, Teixeira FG, Tonoli GHD (2015). Starch/PVA-based nanocomposites reinforced with bamboo nanofibrils. Ind. Crops Prod. 70: 72-83.

Gurunathan T, Mohanty S, Nayak SK (2015). A review of the recent developments in biocomposites based on natural fibres and their application perspectives. Compos Part A-Appl S. 77: 1-25.

Hivechi A, Bahrami SH, Siegel RA, Siehr A, Sahoo A, Milan PB, Joghataei MT, Amoupour M, Simorgh S 

polycaprolactone. Mat Sci Eng. C-Mater. 121: 111855.

444 Hoornaert A, d'Arros C, Heymann MF, Layrolle P (2016). Biocompatibility, resorption and biofunctionality of a new synthetic biodegradable membrane for guided bone regeneration. Biomed. Mater. 11: 045012.

Hoseini SJ, Darroudi M, Oskuee RK, Gholami L, Zak A (2015). Honey-based synthesis of ZnO nanopowders and their cytotoxicity effects. Adv. Powder Technol. 26: 991-996.

Jiang T, Carbone EJ, Lo KWH, Laurencin CT (2015). Electrospinning of polymer nanofibers for tissue regeneration. Prog. Polym. Sci. 46: 1-24.

Jiang LY, Li Y, Xiong CD, Su SP (2017). Preparation and characterization of a novel degradable nano-hydroxyapatite/poly(lactic-co-glycolic) composite reinforced with bamboo fiber. Mat Sci Eng. C-Mater. 75: 1014-1018.

Jiang LY, Li Y, Ma BL, Ding HJ, Su SP, Xiong CD (2018). Effect of Bamboo Fiber Length on Mechanical Properties, Crystallization Behavior, and in Vitro Degradation of Bamboo Fiber/Nanohydroxyapatite/Poly (lactic-co-glycolic) Composite. Ind. Eng. Chem. Res. 57: 4585-4591.

Jiang LY, Ma BL, Li Y, Ding HJ, Su SP, Xiong CD (2019). Effect of bamboo fiber on the degradation behavior and in vitro cytocompatibility of the nano-hydroxyapatite/poly(lactide-co-glycolide) (n-HA/PLGA) composite. Cellulose 26: 1099-1110.

Khalil HPSA, Bhat, IUH, Jawaid M, Zaidon A, Hermawan D, Hadi YS (2012). Bamboo Fibre Reinforced Biocomposites: A Review. Mater Design 42: 353-368.

Kumar A, Negi YS, Choudhary V, Bhardwaj NK (2014). Microstructural and mechanical properties of porous biocomposite scaffolds based on polyvinyl alcohol, nano-hydroxyapatite and cellulose nanocrystals. 
464 Lee BS, Lee CC, Lin HP, Shih WA, Hsieh WL, Lai CH, Takeuchi Y, Chen YW (2016). Functional

465 chitosan membrane with grafted epigallocatechin-3-gallate and lovastatin enhances periodontal

466 tissue regeneration in dogs. Carbohyd. Polym.151: 790-802.

467 Li BW, Liu YH, Zhou YS, You PY, Wang M, Tang L, Deng Y (2020). Development of a novel extracellular matrix membrane with an asymmetric structure for guided bone regeneration. Mater Lett 274: 127926

Li WW, Ma GW, Brazile B, Li N, Dai W, Butler JR, Claude AA, Wertheim JA, Liao J, Wang B (2015). Investigating the Potential of Amnion-Based Scaffolds as a Barrier Membrane for Guided Bone Regeneration. Langmuir 31: 8642-8653.

Li Y, Jiang LY, Xiong CD, Peng WJ (2015). Effect of Different Surface Treatment for Bamboo Fiber on the Crystallization Behavior and Mechanical Property of Bamboo Fiber/Nanohydroxyapatite/Poly(lactic-coglycolic) Composite. Ind. Eng. Chem. Res. 54: 12017-12024.

Liu D, Song J, Anderson DP, Chang PR, Hua Y (2012). Bamboo Fiber and Its Reinforced Composites: Structure and Properties. Cellulose 19: 1449-1480.

Long HB, Wu ZQ, Dong QQ, Shen YT, Zhou WY, Luo Y, Zhang CQ, Dong XM (2019). Effect of polyethylene glycol on mechanical properties of bamboo fiber-reinforced polylactic acid composites. $\mathrm{J}$ Appl Polym Sci 136: 47709.

Ma BL, Jiang LY, Tang CY, Tang S, Su SP, Shu Y(2020). Preparation and properties of biomimetic hydroxyapatite-based nanocomposite utilizing bamboo fiber. Cellulose 27: 2069-2083.

Ma FB, Xia XY, Tang B (2019). Strontium chondroitin sulfate/silk fibroin blend membrane containing microporous structure modulates macrophage responses for guided bone regeneration. Carbohyd. Polym 213: $266-275$. 
Glycerylphytate crosslinker as a potential osteoinductor of chitosan-based systems for guided bone regeneration. Carbohyd. Polym. 213: 266-275.

Muhammad AN, Emel Y, Iskender Y (2017). Intercalated chitosan/hydroxyapatite nanocomposites: Promising materials for bone tissue engineering applications. Carbohyd Polym.175: 38-46.

Niu XL, Wang LF, Xu MJ, Qin M, Zhao LQ, Wei Y, Hu YC, Lian XJ, Liang ZW, Chen S, Chen WY, Huang D (2021). Electrospun polyamide-6/chitosan nanofibers reinforced nano-hydroxyapatite/polyamide-6

Oksana Z, Wu XF, Zhou ZP, Ted A, Jivan T, John H (2020). A Comparative Experimental Study of the Hygroscopic and Mechanical Behaviors of Electrospun Nanofiber Membranes and Solution-cast Films of

Pappu A, Pickering KL, Thakur VK (2019). Manufacturing and characterization of sustainable hybrid

Phuong HAL, Ayob NAI, Blanford CF, Rawi NFM,Szekely G (2019). Nonwoven Membrane Supports from Renewable Resources: Bamboo Fiber Reinforced Poly (Lactic Acid) Composites. ACS Sustain Chem Eng 7: 11885-11893.

Priyadarshini B, Vijayalakshmi U (2018). Development of cerium and silicon co-doped hydroxyapatite nanopowder and its in vitro biological studies for bone regeneration applications. Adv. Powder Technol. 29: 2792-2803.

Prajatelistia E, Sanandiya ND, Nurrochman A, Marseli F, Choy S, Hwang DS (2021). Biomimetic Janus chitin nanofiber membrane for potential guided bone regeneration application. Carbohyd. Polym. 251: 117032.

507 Shakeri A, Khakdan F, Soheili V, Sahebkar A, Rassam G, Asili J (2014). Chemical composition, antibacterial 

58: $315-321$

Tang S, Jiang LY, Ma BL, Tang CY, Wen Y, Zhang N, Zhang Y, Su SP (2020). Preparation and characterization of bamboo fiber/chitosan/nano-hydroxyapatite composite membrane by ionic crosslinking. Cellulose 27: 5089-5100.

513 Weng RG, Huang X, Liao DQ, Xu S, Peng L, Liu XZ (2020). A novel cellulose/chitosan composite 514 nanofiltration membrane prepared with piperazine and trimesoyl chloride by interfacial polymerization. RSC Adv 10: 1309-1318.

Yadav S, Gangwar S (2020). The effectiveness of functionalized nano-hydroxyapatite filler on the physical and mechanicalproperties of novel dental restorative composite. Cellulose 69: 907-918.

518 Ye HL, Zhu JJ, Deng D, Jin SE, Li JD, Man Y (2019). Enhanced osteogenesis and angiogenesis by $519 \mathrm{PCL} /$ chitosan/Sr-doped calcium phosphate electrospun nanocomposite membrane for guided bone. J Biomat 520 Sci-Polym E 30: 1505-1522.

521 Yu L, Cai Y, Wang H, Pan LB, Li JY, Chen S, Liu Z, Han FX, Li B (2020). 522 Biomimetic bone regeneration using angle-ply collagen membrane-supported cell sheets subjected to mechanical conditioning. Acta Biomater 112: 75-86.

Zhao XJ, Zhou LY, Li QD, Zou QX, Du C (2018). Biomimetic mineralization of carboxymethyl chitosan nanofibers with improved osteogenic activity in vitro and in vivo. Carbohyd Polym.195: 225-234.

Zhu J, Tang D, Lu ZH, Xin ZY, Song J, Meng JM, Lu JR, Li Z, Li JS (2020). Ultrafast bone-like apatite formation on highly porous poly(l-lactic acid)-hydroxyapatite fibres. Mat Sci Eng. C-Mater. 116, 11168.

528 Zuo YF, Li WH, Li P, Liu WJ, Li XG, Wu YQ (2018). Preparation and characterization of polylactic acid-g-bamboo fiber based on in-situ solid phase polymerization. Ind. Crops Prod. 123: 646-653. 
532 In this manuscript, bamboo fiber $(\mathrm{BF})$ was firstly chosen to prepare nano-hydroxyapatite/BF 533 (n-HA/BF) composite membrane replacing other polymers by casting method, and the membrane

534 forming mechanism and the effects of different forming membrane methods, drying methods and 535 n-HA amounts on the corresponding n-HA/BF membrane were investigated. Results 536 demonstrated that n-HA dispersity in BF matix was not affected by the prepartion condition, 537 however, the morphologies of membrane was determined by the different preparation conditions 538 owing to different hydrogen bond shrinkage. Moreover, the hydrophilicity of the composite 539 membrane was improved under the condition of the membrane formation in oven, freeze drying 540 and high addition content of n-HA, and the mechanical properties of composite membrane 541 depended on n-HA content. In vitro soaking behavior indicated that the degradability and

542 bone-like apatite deposition could be controled by different preparation conditions. And the cell 543 proliferation experiment showed that the n-HA/BF composite membranes obtained under 544 different preparation conditions were all non-toxic. The above results indicated that the n-HA/BF 545 composite membrane could be obtained by a simple casting technique, and the properties could 546 be controlled by adopting different preparation conditions, which would have a great promising 547 as guide bone tissue regeneration (GBR) membrane, and the study would provide a new 548 application for BF in biomedical field.

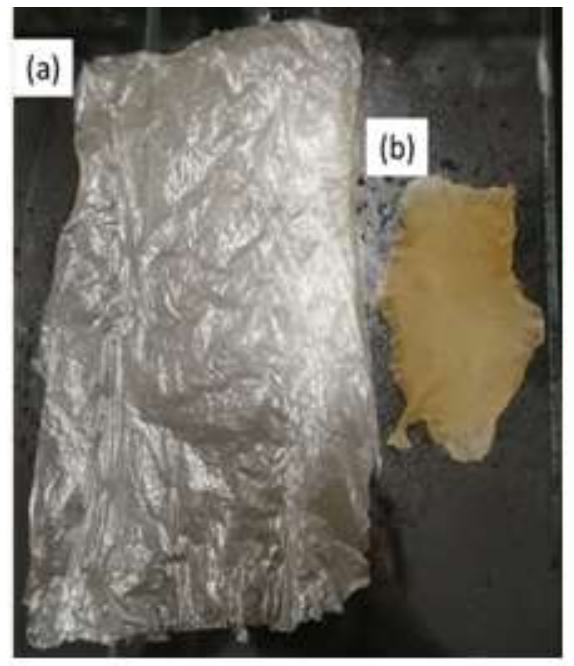

Membrane appearances formed by different conditions. (a) in oven, (b) in air.
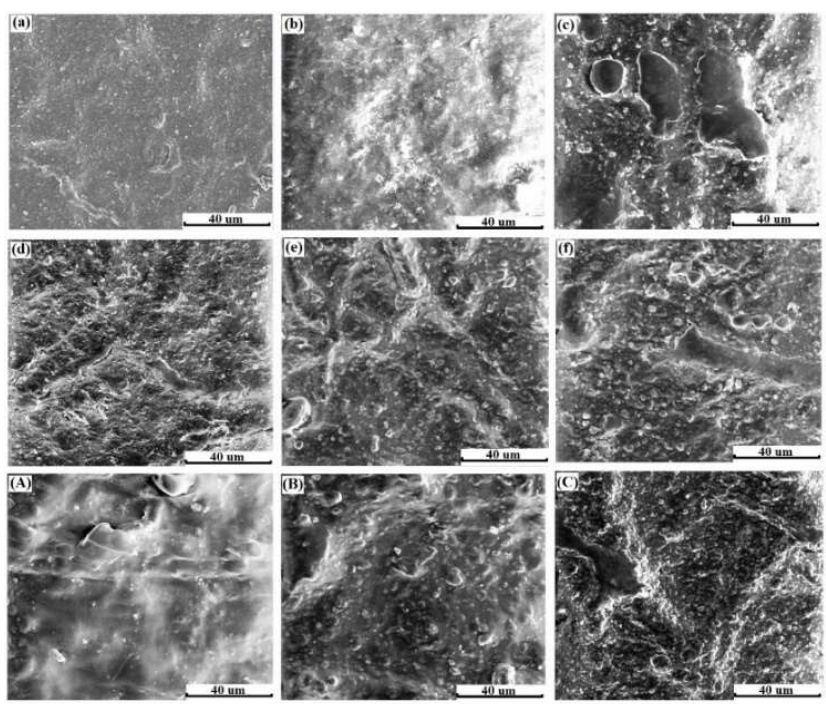

SEM micrographs of samples. (a)Air-Air, (b)Air-oven, (c)Air-freeze drying, (d)Oven-air, (e)Oven-oven, (f)Oven-freeze 24 drying, (A)10\%HA/BF, (B) 30\%HA/BF, (C)40\%HA/BF. 